\title{
A New Mode of Encryption Providing a Tweakable Strong Pseudo-random Permutation
}

\author{
Debrup Chakraborty and Palash Sarkar \\ Applied Statistics Unit \\ Indian Statistical Institute \\ 203 B.T. Road \\ Kolkata 700108, India \\ \{debrup_r, palash\}@isical.ac.in
}

\begin{abstract}
We present PEP, which is a new construction of a tweakable strong pseudo-random permutation. PEP uses a hash-encrypt-hash approach which has been recently used in the construction of HCTR. This approach is different from the encrypt-mask-encrypt approach of constructions such as CMC, EME and EME* . The general hash-encrypthash approach was earlier used by Naor-Reingold to provide a generic construction technique for an SPRP (but not a tweakable SPRP). PEP can be seen as the development of the Naor-Reingold approach into a fully specified mode of operation with a concrete security reduction for a tweakable strong pseudo-random permutation. HCTR is also based on the Naor-Reingold approach but its security bound is weaker than PEP. Compared to previous known constructions, PEP is the only known construction of tweakable SPRP which uses a single key, is efficiently parallelizable and can handle an arbitrary number of blocks.
\end{abstract}

Keywords: mode of operation, tweakable encryption, strong pseudorandom permutation.

\section{Introduction}

A block cipher is a fundamental primitive in cryptography. A block cipher by itself can encrypt only fixed length strings. Applications in general require encryption of long and arbitrary length strings. A mode of operation of a block cipher is used to extend the domain of applicability from fixed length strings to long and variable length strings. The mode of operation must be secure in the sense that if the underlying block cipher satisfies a certain notion of security, then the extended domain mode of operation also satisfies an appropriate notion of security.

A formal model of security for a block cipher is a pseudo-random permutation 9] which is formalized as a keyed family of permutations. Pseudo-randomness of the permutation family requires a computationally bounded adversary to be unable to distinguish between a random permutation and a permutation picked at random from the family. Strong pseudo-random permutations (SPRPs) 
require computational indistinguishability even when the adversary has access to the inverse permutation.

A mode of encryption usually provides two security assurances - privacy and authenticity. For example, OCB [14] is a mode of operation (providing both privacy and authenticity) which extends the domain of a block-cipher to arbitrary strings. An SPRP which can encrypt arbitrary length strings, can be viewed as a mode of operation with a somewhat different goal. Such a mode of operation is length preserving and no tag is produced. Hence, authentication is of limited nature. A change in the ciphertext cannot be detected but the decryption of the tampered ciphertext will result in a plaintext which is indistinguishable from a random string. Additional redundancy in the message introduced by higher level applications might even allow the detection of the tampering. This point is discussed in details by Bellare and Rogaway [1.

Tweakable encryption was introduced by Liskov, Rivest and Wagner [8] which added an extra input called tweak to a block cipher. This allows simplification of several applications. The paper 8 introduced both tweakable PRP and SPRP. In the adversarial model for tweakable SPRP, the adversary queries the encryption (resp. decryption) oracle with a tweak and the plaintext (resp. ciphertext). The adversary is allowed to repeat the tweaks in its queries to the encryption and the decryption oracles. A tweakable SPRP provides a mode of operation having all the advantages of an SPRP with the additional flexibility of having a tweak. The constructions CMC [4, EME [5, EME* 3] and HCTR [7] are proved to be tweakable SPRPs under the assumption that the underlying block cipher is an SPRP. As mentioned in [4], such a primitive is well suited for disk encryption where the tweak can be considered to be the sector address.

Our Contributions: We present a new construction of a tweakable SPRP called PEP (for Polynomial hash-Encrypt-Polynomial hash). PEP uses a block cipher which can encrypt an $n$-bit string to construct an encryption algorithm which can encrypt $m n$-bit strings for any $m \geq 1$. It uses two Wegman-Carter [16 style polynomial hashes over the binary field $G F\left(2^{n}\right)$. (Similar hashes have been earlier used in GCM [10] and HCTR.) The new construction is proved to be a tweakable SPRP assuming that the underlying block cipher is an SPRP. Below we mention some interesting features of PEP.

1. PEP is a fully specified mode of operation providing a tweakable SPRP. The security proof for PEP provides a concrete security bound which is the usual quadratic birthday bound earlier obtained for CMC, EME and EME*. The security bound of HCTR is weaker and the security degradation is cubic.

2. The total computation cost of PEP for encrypting an $m$-block message consists of $m+5$ block cipher calls for $m \geq 2(m+3$ for $m=1)$, and $(4 m-6)$ multiplications in $G F\left(2^{n}\right)$. In contrast, CMC requires $2 m+1$ block cipher calls; EME* requires $(2 m+m / n+1)$ block cipher calls; and HCTR requires $m$ block cipher calls and $2(m+1)$ many $G F\left(2^{n}\right)$ multiplications. The exact comparison of the computation costs between PEP and CMC depends upon several factors such as the implementation platform (hardware or software), availability of suitable co-processors (for software implementation), 
availability of parallel encryption blocks (for hardware implementation) and most importantly on the actual block cipher being used and the efficiency of its implementation.

3. Currently, PEP fills a gap in the known constructions. It is the only known construction of tweakable SPRP which uses a single key, is efficiently parallelizable and can handle arbitrary number of message blocks. Table 1 in Section 3 provides a detailed comparison of PEP with the other tweakable SPRPs.

Related Constructions: To the best of our knowledge, the first suggestion for constructing an SPRP was made by Naor and Reingold in [11. They suggested the hash-encrypt-hash approach used in PEP. However, as discussed in [4, the description in [11 is at a top level and also the later work 12 does not fully specify a mode of operation. Perhaps more importantly, the work [12] does not consider tweakable SPRP since it predates the introduction of tweakable primitives in [8].

The NR approach was rejected in [4] as not being capable of efficient instantiation. The work in [4] and also the later constructions [5]3] follow a encrypt-maskencrypt strategy, i.e., there are two layers of encryptions with a layer of masking in between. CMC [4 provides the first efficient, fully specified construction of a tweakable SPRP. Parallel versions of the encrypt-mask-encrypt strategy have been proposed as EME and EME*.

Interestingly, the NR approach made a recent comeback in the HCTR construction. The HCTR construction combines the NR type invertible hash functions with the counter mode of operation. This results in an efficient tweakable SPRP which can handle any message having length $\geq n$ bits. The drawback of HCTR is its weaker security bound and the requirement of having two keys. Currently, PEP can be viewed as the development of the NR approach to the construction of tweakable SPRP.

\section{Specification of PEP}

We construct the tweakable enciphering scheme PEP from a block cipher $E$ : $\mathcal{K} \times\{0,1\}^{n} \rightarrow\{0,1\}^{n}$ and call it $\mathrm{PEP}[E]$. The key space of $\mathrm{PEP}[E]$ is same as that of the underlying block cipher $E$ and the tweak space is $\mathcal{T}=\{0,1\}^{n}$. The message space consists of all binary strings of size $m n$ where $m \geq 1$.

Finite Field Arithmetic: An $n$-bit string can also be viewed as an element in $G F\left(2^{n}\right)$. Thus, we will consider each $n$-bit string in the specification of PEP as a polynomial over $G F(2)$ modulo a fixed primitive polynomial $\tau(x)$ of degree $n$. For each $n$-bit string $Z$ that occur in the description of PEP, we will use $Z(x)$ to denote the corresponding polynomial in $G F\left(2^{n}\right)$. The expressions $p(x) M_{1}$ (resp. $x E N)$, represent the $n$-bit string obtained by multiplying the polynomials $p(x)$ and $M_{1}$ (resp. $x$ and $E N$ ) modulo $\tau(x)$. Also for two $n$-bit strings $Z_{1}$ and $Z_{2}$, the expression $Z_{1}(x) Z_{2}(x)$ denotes the $n$-bit string obtained by multiplying $Z_{1}(x)$ 
and $Z_{2}(x)$ modulo $\tau(x)$. Finally, by $R^{-1}(x)$ we will denote the multiplicative inverse of $R(x)$ modulo $\tau(x)$ when $R(x) \neq 0$.

Definition 1. Let $m \geq 3$ and $p_{m, 1}(x), \ldots, p_{m, m}(x)$ be a sequence of polynomials over $G F(2)$ each having degree at most $n-1$. We call such a sequence an allowed sequence with respect to a primitive polynomial $\tau(x)$ of degree $n$ if the following two conditions hold.

1. $\bigoplus_{i=1}^{m} p_{m, i}(x) \equiv 0 \bmod \tau(x)$.

2. For $1 \leq i<j \leq m,\left(p_{m, i}(x) \oplus p_{m, j}(x)\right) \not \equiv 0 \bmod \tau(x)$.

From the definition, it is clear that for an allowed sequence to exist, we must have $m \geq 3$. The parameter $m$ in the specification of PEP will represent the number of blocks to be encrypted (or decrypted). Later we show how to easily define such an allowed sequence. Since $\tau(x)$ is fixed, we will simply write "allowed sequence" instead of "allowed sequence with respect to $\tau(x)$ ".

The notation $\operatorname{bin}(m)$ denotes the $n$-bit binary representation of the integer $m$. For example, $\operatorname{bin}(1)=0^{n-1} 1$ and $\operatorname{bin}(2)=0^{n-2} 10$. The specification of PEP consists of three cases: $m=1 ; m=2$; and $m \geq 3$. The cases $m=1$ and $m=2$ are shown in Figure 2, Figure 3 shows the encryption of a 4-block message. The complete encryption and decryption algorithms are shown in Figure 1.

Remark: For decryption to be possible, we need $R(x)$ to have a multiplicative inverse modulo $\tau(x)$. Since $R(x)$ is a polynomial of degree at most $n-1$, the only value for which $R(x)$ does not have such an inverse is $R(x)=0$. For such an $R$, the protocol is not defined. Note that $R=E_{K}(T)$. Assuming $E_{K}()$ to be a random permutation, the probability $R=0$ is $1 / 2^{n}$. Since $n \geq 128$, the probability of getting a $T$ for which the protocol is not defined is negligible.

Basic intuition behind the construction: The basic idea of the construction is to compute a polynomial hash (Wegman-Carter [16]) of the message. (Similar hashes are used in the GCM mode of operation [10] and HCTR.) This hash is XOR-ed with $E N$ to obtain the element $M P P$ whose expression is the following.

$$
M P P=E N \oplus \bigoplus_{i=1}^{m} P_{i}(x) R^{i-1}(x) .
$$

The mask $M_{1}$ is obtained by encrypting $M P P$. Since we are aiming at an SPRP, we should ensure that each ciphertext bit depends upon all the plaintext bits. To do this, the mask $M_{1}$ is "mixed" to the message blocks to obtain the $P P P_{i}$ 's. While doing this we must be careful. During decryption, we will obtain the $P P P_{i}$ 's after the decryption layer. Thus, we should be able to compute $M P P$ from the $P P P_{i}$ 's. To ensure that this can be done, we do two things. First we convert the $P_{i}$ 's to $P P_{i}$ 's by multiplying with $R^{i-1}$. The second thing is to "distribute" $M_{1}$ among the $P P_{i}$ 's while obtaining the $P P P_{i}$ 's so as to ensure that

$$
\bigoplus_{i=1}^{m} P P P_{i}=\bigoplus_{i=1}^{m} P P_{i}=\bigoplus_{i=1}^{m} P_{i}(x) R^{i-1}(x)=M P P \oplus E N
$$


This follows from the first property of allowed sequences, namely, $\bigoplus_{i=1}^{m} p_{m, i}(x) \equiv$ $0 \bmod \tau(x)$ and hence $M P P$ can be computed either as $\bigoplus_{i=1}^{m} P P_{i} \oplus E N$ or as $\bigoplus_{i=1}^{m} P P P_{i} \oplus E N$. Since allowed sequences exist only for $m \geq 3$, we need to tackle the cases $m=1$ and $m=2$ separately. In the case $m=1$, there is no requirement to "distribute" $M_{1}$ among the message blocks while in the case of $m=2$, this is a bit tricky to do. In the last case, we distribute $M_{1}$ as $M_{1} \oplus E N$ and $M_{1} \oplus E E N$. Note that the XOR of these two elements is $E N \oplus E E N$ (and not 0 ). However, both $E N$ and $E E N$ can be computed from the tweak and the length of the message and hence $M P P=P P_{1} \oplus P P_{2} \oplus E N=P P P_{1} \oplus P P P_{2} \oplus E E N$ and can be computed both during encryption and decryption.

The computation after the encryption layer is similar to the computation before the encryption layer. This is because we are constructing an SPRP and the view from the decryption end will be similar to the view from the encryption end. In the decryption query, we work with $L(x)=R^{-1}(x)$ while computing the polynomial hash. This is required to ensure the consistency of decryption. This leads to decryption requiring one extra inversion operation making it slightly more costlier than encryption.

\subsection{Construction of Allowed Sequence of Polynomials}

In this section, we provide one construction of allowed sequence. We do not claim this to be the only possibility; there may be others.

Let $\tau(x)$ be a primitive polynomial of degree $n$. Let $m_{\max }$ be a positive integer such that $\tau(x)$ does not divide any trinomial of degree less than $m_{\max }$. Estimates of $m_{\max }$ have been studied in the context of attacks on the nonlinear combiner model for stream ciphers [7. (To use a primitive polynomial $\tau(x)$ in such stream ciphers, it is necessary that $\tau(x)$ does not divide a low degree trinomial.) This study indicates that $m_{\max }$ is around $2^{n / 3}$. Given $\tau(x)$, there are algorithms for computing sparse multiples of $\tau(x)$. See [15] for a discussion on this issue.

We will be constructing an allowed sequence of length $m_{\max }$ and hence we will not be able to encrypt a message having more than $m_{\max }$ blocks. For $n=128$, we have $2^{n / 3} \approx 2^{42.6}$ and hence $m_{\max }$ is also around $2^{42.6}$. The ability to encrypt messages having at most $2^{42.6}$ many blocks is sufficient for all practical purposes.

Let $m=3 t \leq m_{\max }$, with $t \geq 1$. We define a sequence of polynomials $\tau_{3 t, 1}(x), \ldots, \tau_{3 t, 3 t}(x)$ in the following manner.

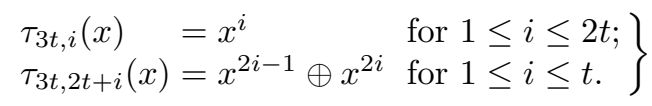

Let $3 \leq m \leq m_{\max }$. We define a sequence of polynomials $p_{m, 1}(x), \ldots, p_{m, m}(x)$ in the following manner.

$m=3 t:$ Define $p_{m, i}(x)=\tau_{m, i}(x)$.

$m=3 t+1:$ Define $p_{m, 1}=1 \oplus x, p_{m, 2}(x)=x \oplus x^{2}, p_{m, 3}(x)=x^{2} \oplus x^{3}$, $p_{m, 4}(x)=x^{3} \oplus 1$ and $p_{m, 4+i}(x)=x^{3} \tau_{3(t-1), i}(x)$, for $1 \leq i \leq m$. 


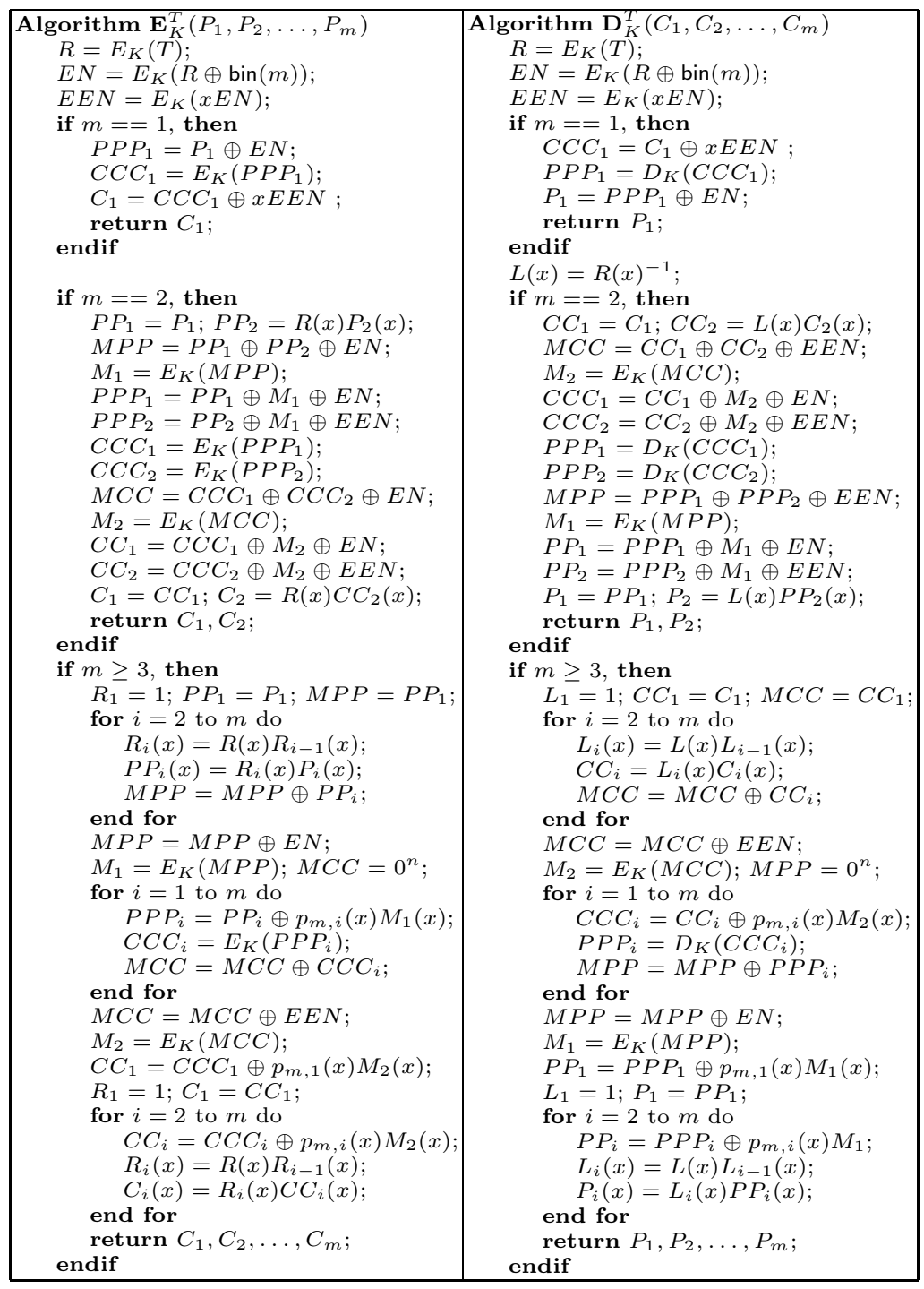

Fig. 1. Encryption and Decryption using PEP

$m=3 t+2:$ Define $p_{m, 1}=1 \oplus x, p_{m, 2}(x)=x \oplus x^{2}, p_{m, 3}(x)=x^{2} \oplus x^{3}$, $p_{m, 4}(x)=x^{3} \oplus x^{4}, p_{m, 5}(x)=x^{4} \oplus 1$ and $p_{m, 5+i}(x)=x^{4} \tau_{3(t-1), i}(x)$, for $1 \leq i \leq m$.

From the definition, note that for $m_{1} \neq m_{2}$, we may have $p_{m_{1}, i}(x) \neq p_{m_{2}, i}(x)$. Later we show that due to its simple form, multiplication by $p_{m, i}(x)$ is quite efficient.

Proposition 1. The sequence of polynomials $p_{m, 1}(x), \ldots, p_{m, m}(x)$ is an allowed sequence. 


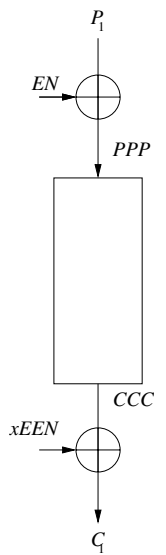

(a)

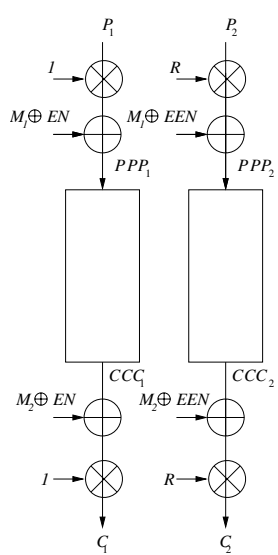

(b)

Fig. 2. (a) Enciphering one plaintext block with PEP, (b) Enciphering two plaintext blocks with PEP

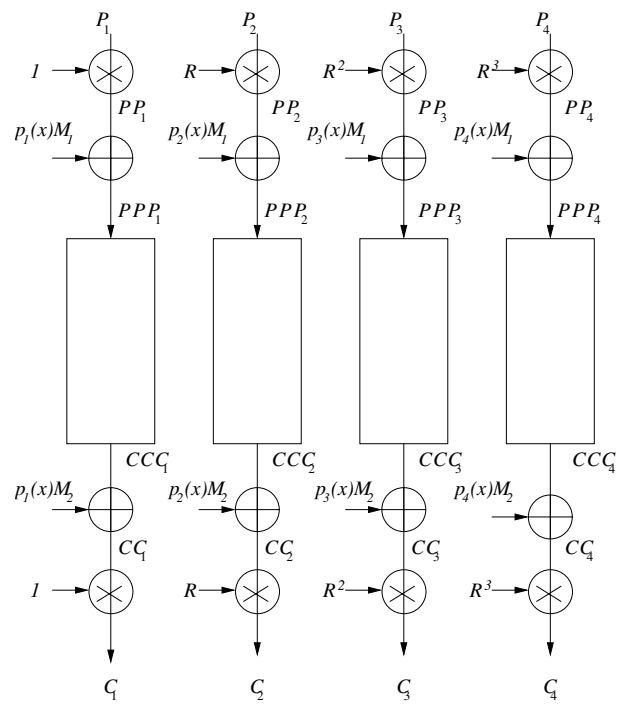

Fig. 3. Enciphering four plaintext blocks with PEP. $R=E_{K}(T), E N=E_{K}(R \oplus$ $\left.\operatorname{bin}_{n}(4)\right), E E N=E_{K}(x E N), M_{1}=E_{K}\left(\bigoplus_{i=1}^{4} R^{i-1}(x) P P_{i}(x) \oplus E N\right), M_{2}=$ $E_{K}\left(\bigoplus_{i=1}^{4} C C C_{i} \oplus E E N\right)$.

Proof : From the definition of $\tau_{3 t, i}(x)$ it is easy to verify that $\bigoplus_{i=1}^{3 t} \tau_{3 t, i}(x)=0$. From this it is easy to see that $\bigoplus_{i=1}^{m} p_{m, i}(x)=0$. This establishes the first condition for allowed sequences.

For the second condition, we must show that $p_{m, i}(x) \oplus p_{m, j}(x) \not \equiv 0 \bmod \tau(x)$. There are three cases to consider. 
Both $p_{m, i}(x)$ and $p_{m, j}(x)$ are monomials: By construction the degrees of both $p_{m, i}(x)$ and $p_{m, j}(x)$ are at most $m_{\max }<2^{n}-1$. Hence, by the primitivity of $\tau(x)$ we have the required condition.

Both $p_{m, i}(x)$ and $p_{m, j}(x)$ are 2-nomials: By construction $p_{m, i}(x)=x^{i_{1}} \oplus x^{i_{1}+1}$ and $p_{m, j}(x)=x^{j_{1}} \oplus x^{j_{1}+1}$. Assume without loss of generality that $i_{1}<j_{1}$. Then, $p_{m, i}(x) \oplus p_{m, j}(x)=x^{i_{1}}(1 \oplus x)\left(1 \oplus x^{j_{1}-i_{1}}\right)$. Again, the primitivity of $\tau(x)$ ensures the required condition.

One of $p_{m, i}(x)$ and $p_{m, j}(x)$ is a monomial and the other is a 2-nomial: In this case, $p_{m, i}(x) \oplus p_{m, j}(x)$ is a trinomial of degree at most $m_{\max }$. The definition of $m_{\max }$ ensures the required condition.

This completes the proof.

\subsection{Computation of $p_{m, i}(x) M_{1}(x)$}

In the encryption and decryption algorithms, we need to compute $p_{m, i}(x) M_{1}(x)$ and $p_{m, i}(x) M_{2}(x)$. We show how these may be efficiently computed. For this it is sufficient to show how to efficiently compute $\tau_{3 t, i}(x) M_{1}$ and $\tau_{3 t, i}(x) M_{2}$.

The polynomials $\tau_{3 t, i}(x)$ satisfy the following recurrences:

$$
\begin{aligned}
& \tau_{3 t, i}(x)=x \tau_{3 t, i-1}(x) \quad \text { for } 2 \leq i \leq 2 t \\
& \tau_{3 t, 2 t+1}=\tau_{3 t, 1}(x) \oplus \tau_{3 t, 2}(x) ; \\
& \tau_{3 t, i}(x)=x^{2} \tau_{3 t, i-1}(x) \quad \text { for } 2 t+2 \leq i \leq 3 t .
\end{aligned}
$$

Define $M_{1,3 t, i}=\tau_{3 t, i}(x) M_{1}$. Then using the above recurrences, we have

$$
\begin{aligned}
& M_{1,3 t, i}(x)=x M_{1,3 t, i-1} \text { for } 2 \leq i \leq 2 t \\
& M_{1,3 t, 2 t+1}=M_{1,3 t, 1}(x) \oplus M_{1,3 t, 2} \\
& M_{1,3 t, i}(x)=x^{2} M_{1,3 t, i-1} \text { for } 2 t+2 \leq i \leq 3 t .
\end{aligned}
$$

Using these recurrences, it is easy to compute all the $\tau_{3 t, i}(x) M_{1}$ 's; the requirement is to multiply by either $x$ or $x^{2}$ and perform a bitwise XOR. Multiplying by $x$ and $x^{2}$ is much more efficient than a general multiplication modulo $\tau(x)$. A similar computation will yield the products $\tau_{3 t, i}(x) M_{2}$.

\section{Features of PEP}

Here we discuss some of the important features and limitations of PEP.

Message Length: PEP does not produce any ciphertext expansion as it does not produce any tag. The tweak is not considered to be a part of the ciphertext. This is similar to CMC, EME, EME* and HCTR. The current version of PEP can only handle messages whose length is a multiple of $n$. This is similar to CMC. EME can handle messages of lengths $m n$, with $1 \leq m \leq n$, while EME* and HCTR can handle messages of lengths $\geq n$. Modification of PEP to handle messages of lengths $\geq n$ is a future task. 
There is a (theoretical) restriction on the number of blocks in a particular message to be encrypted by PEP. The number of blocks in any one message to be encrypted by PEP is at most $m_{\max }$. For $n=128$, this implies that a single message can contain at most around $2^{42.6}$ blocks, which is sufficient for all practical purposes (see Section 2.1).

Single Block Cipher Key: PEP uses the same key for all the block cipher calls. $\mathrm{CMC}$ and EME require a single key. On the other hand, EME* requires three keys and HCTR requires two keys. A single block cipher key saves key storage space and key setup costs.

Tweak: Encryption under PEP requires an $n$-bit tweak. The tweak need not be random, unpredictable or secret. The adversary is allowed to repeat a tweak in queries to the encryption and decryption oracles. The tweak is required for decryption and hence has to be available at both the receiver and the sender ends. This may be achieved by maintaining a shared counter between the two parties or it may be such that it can be understood from the context. An example of the later is the sector address in disk encryption applications.

Online/Offline: An encryption scheme is called online if it can output a stream of ciphertext bits as a stream of plaintext bits arrive. PEP is not online. PEP incorporates the effect of the whole plaintext on each ciphertext bit. Hence, construction of PEP does not allow it to output a single block of ciphertext unless it has seen the total message. We note that no construction of SPRP (tweakable or otherwise) can be online for the same reason as PEP.

Consider the encryption algorithm of PEP for $m \geq 3$. The algorithm consists of three separate for loops. The first loop computes the $P P_{i}$ 's and the quantity $\bigoplus_{i=1}^{m} R_{i}(x) P_{i}(x)$. The values of the $P P_{i}$ 's need to be stored for use by the second loop. The second loop computes the $P P P_{i}$ 's and the $C C C_{i}$ 's and also the quantity $\bigoplus_{i=1}^{m} C C C_{i}$. The $P P P_{i}$ 's do not need to be stored but the value of the $C C C_{i}$ 's need to be stored for use by the third loop. The third loop produces the $C C_{i}$ 's and the $C_{i}$ 's which completes the encryption. (Decryption also has a similar structure).

To summarize, the algorithm makes a pass over the $P_{i}$ 's to produce the $P P_{i}$ 's which are stored; makes a pass over the $P P_{i}$ 's to produce the $C C C_{i}$ 's which are also stored; and finally makes a pass over the $C C C_{i}$ 's to produce the $C_{i}$ 's. This makes it a three pass algorithm. Note that the $P P_{i}$ 's can be written over the $P_{i}$ 's and the $C C C_{i}$ 's can be written over the $P P_{i}$ 's. Thus, the intermediate quantities $P P_{i}$ 's and the $C C C_{i}$ 's do not require any extra storage.

HCTR is also a hash-encrypt-hash type construction and requires three passes for reasons similar to that of PEP. The algorithmic descriptions of CMC, EME and $\mathrm{EME}^{*}$ as given in the respective papers suggest these algorithms to be threepass algorithms. On the other hand, a careful consideration of the algorithms show that all of these algorithms can be implemented using two passes over the data. Basically, these algorithms are of the form encrypt-mask-encrypt. The first encryption layer needs to be completed in one pass over the data. Then 
the mask is computed. The actual masking of the intermediate values and the second encryption layer can be combined in a single pass.

Note that any algorithm requiring more than one pass cannot be online and also at least one set of intermediate variables need to be stored. If we overwrite the $P_{i}$ 's then no extra storage is required for either two or three pass algorithms. On the other hand, if we wish to preserve the $P_{i}$ 's, then the same amount of extra space is required by both two and three pass algorithms. Further, a two pass algorithm is not necessarily more efficient than a three pass algorithm. We have to compare the total amount of computation done by the two algorithms to determine relative efficiency. We consider this issue next.

Computation Cost: PEP performs two polynomial hashes and one layer of block cipher encryption. The total number of block cipher encryptions for an $m$-block message is 4 if $m=1$; and is $m+5$ if $m \geq 2$. The polynomial hashes are used to compute $M P P$ and $M C C$ for $m \geq 2$, the two computations being similar.

To compute $M P P$ we have to compute $\bigoplus_{i=1}^{m} P_{i}(x) R^{i-1}(x)$. Using Horner's rule this can be computed using $(m-1)$ multiplications over $G F\left(2^{n}\right)$. But Horner's rule does not compute the values $P P_{i}=P_{i}(x) R^{i-1}(x)$. Since the values of $P P_{i}$ 's are also required, using Horner's rule does not help. We have to compute $R^{2}(x), \ldots, R^{m-1}(x)$ and $P_{2}(x) R(x), \ldots, P_{m} R^{m-1}(x)$. These require a total of $2 m-3$ multiplications in $G F\left(2^{n}\right)$. Similarly, a total of $2 m-3$ multiplications are required for computing $M C C$ and the $C C_{i}$ 's. Hence, for $m \geq 2$, PEP requires a total of $4 m-6$ finite field multiplications. In addition, there are the multiplications of the type $p_{m, i}(x) M_{1}$ and $p_{m, i}(x) M_{2}$. But as discussed in Section 2.2, these can be computed very efficiently.

If sufficient memory is available, then the values $R^{2}(x), \ldots, R^{m-1}(x)$ computed during the computation of $M P P$ and the $P P_{i}$ 's can be stored and used during the computation of $M C C$ and the $C C_{i}$ 's. (This can also be combined with the parallel computation strategy discussed below.) This brings down the total number of multiplications to $3 m-4$.

We note that decryption requires the computation of one finite field inverse. The cost of this is amortized over the entire computation and will not reflect on the overall cost if $m$ is moderately large. The main cost will be that for hardware implementation since we will have to implement a finite field inverter requiring more chip area.

Parallelism: The encryption layer is fully parallelizable though the computations of $R, E N, E E N, M_{1}$ and $M_{2}$ has to be sequential. Thus, for $m \geq 2$, we require at least six parallel encryption rounds irrespective of the number of available block cipher units - five for computing the above quantities and at least one for encrypting the $P P P_{i}$ 's.

The computation of the $P P_{i}$ 's and $M P P$ can be parallelized in the following manner. We illustrate by an example. Suppose there are 4 finite field multipliers available. In the first step, the quantity $R^{2}$ is computed. Now consider the following parallel schedule for the four multipliers. 


\begin{tabular}{|l|c|c|c|c|}
\hline & multiplier 1 & multiplier 2 & multiplier 3 & multiplier 4 \\
\hline Round 1 & $\left(P_{2}(x) * R(x)\right)$ & $\left(P_{3}(x) * R^{2}(x)\right)$ & $\left(R(x) * R^{2}(x)\right)$ & $\left(R^{2}(x) * R^{2}(x)\right)$ \\
\hline Round 2 & $\left(P_{4}(x) * R^{3}(x)\right)$ & $\left(P_{5}(x) * R^{4}(x)\right)$ & $\left(R^{3}(x) * R^{2}(x)\right)$ & $\left(R^{4}(x) * R^{2}(x)\right)$ \\
\hline Round 3 & $\left(P_{6}(x) * R^{5}(x)\right)$ & $\left(P_{7}(x) * R^{6}(x)\right)$ & $\left(R^{5}(x) * R^{2}(x)\right)$ & $\left(R^{6}(x) * R^{2}(x)\right)$ \\
\hline$\ldots$ & $\ldots$ & $\ldots$ & $\ldots$ & $\ldots$ \\
\hline
\end{tabular}

Using this schedule, all the four multipliers can be kept busy in all the rounds (except possibly for the last round and the initial computation of $R^{2}$ ). A similar schedule can be built for computing $M C C$ and the $C C_{i}$ 's. In general using $\kappa$ many multipliers, all the multiplications can be completed in approximately $\lceil(4 m-6) / \kappa\rceil$ many parallel rounds which is optimal for $\kappa$ many multipliers.

HCTR uses a polynomial hash which can be evaluated by Horner's rule. On the other hand, there is no straightforward way of parallelizing the polynomial computation without increasing the total number of multiplications. The approach described above can be used to obtain parallel implementation of polynomial evaluation and this would approximately double the number of multiplications required in evaluating using Horner's rule.

Provable Security: PEP is provably secure. We state a theorem related to the security of PEP in Section 4 and provide the proof ideas (for the full proof see [2]). The concrete security bound that we obtain for PEP is similar to that obtained for CMC, EME and $\mathrm{EME}^{*}$ and is as expected for a mode of operation. Loosely speaking, the theorem shows that the advantage of an adversary in attacking $\operatorname{PEP}[E]$ as a tweakable SPRP is bounded above by the advantage of an adversary in attacking $E$ as an SPRP plus an additive factor which is approximately equal to $c \sigma_{n}^{2} / 2^{n}$, where $c$ is a constant and $\sigma_{n}$ is the total number of blocks (plaintext or ciphertext) provided by the adversary in its queries to the encryption and the decryption oracles. The security bound of HCTR is considerably weaker than the other modes of operations including PEP. For HCTR, the quantity $c \sigma_{n}^{2} / 2^{n}$ is replaced by $c \sigma_{n}^{3} / 2^{n}$, i.e., there is a cubic security degradation rather than the usual quadratic degradation. One consequence of a weaker security bound is that the secret keys need to changed much earlier compared to the other modes.

Comparison: Table 1 provides a comparison of the various features of $\mathrm{PEP}$ and other modes of operations which are SPRPs. We make the following points based on Table 1 .

HCTR is the only previously known fully specified tweakable SPRP which is of the hash-encrypt-hash type. The NR construction is too incomplete (and also not tweakable) to permit a proper comparison to other constructions. The main drawback of HCTR is its weaker security bound and the requirement of two keys. It has other good features such as ability to handle all message lengths $\geq n$, and lower computation cost. The encryption layer of HCTR is fully parallel. The two hash layers can be implemented in parallel with computation cost similar to that of PEP.

PEP is currently the only known single key, efficiently parallelizable algorithm which can handle arbitrary number of $n$-bit blocks. CMC, EME* and HCTR can 
Table 1. Comparison of SPRPs using an $n$-bit block cipher, an $n$-bit tweak and for $m$ message blocks. (We assume $m \geq 3$.) [BC]: one block cipher invocation; $[\mathrm{M}]$ : one $G F\left(2^{n}\right)$ multiplication.

\begin{tabular}{|c||c|c|c|c|c|c|c|}
\hline Mode & sec. bnd. & $\begin{array}{c}\text { computation } \\
\text { cost }\end{array}$ & keys & msg. len. & passes & $\begin{array}{c}\text { enc. } \\
\text { layers }\end{array}$ & parallel? \\
\hline \hline CMC & $\sigma_{n}^{2} / 2^{n}$ & $(2 m+1)[\mathrm{BC}]$ & 1 & $m n, m \geq 1$ & 2 & 2 & No \\
\hline EME & $\sigma_{n}^{2} / 2^{n}$ & $(2 m+2)[\mathrm{BC}]$ & 1 & $m n, 1 \leq m \leq n$ & 2 & 2 & Yes \\
\hline EME $^{*}$ & $\sigma_{n}^{2} / 2^{n}$ & $\left(2 m+\frac{m}{n}+1\right)[\mathrm{BC}]$ & 3 & $\geq n$ & 2 & 2 & Yes \\
\hline HCTR & $\sigma_{n}^{3} / 2^{n}$ & $\begin{array}{c}m[\mathrm{BC}] \\
+2(m+1)[\mathrm{M}]\end{array}$ & 2 & $\geq n$ & 3 & 1 & partial \\
\hline \hline PEP & $\sigma_{n}^{2} / 2^{n}$ & $\begin{array}{c}(m+5)[\mathrm{BC}] \\
+(4 m-6)[\mathrm{M}]\end{array}$ & 1 & $m n, m \geq 1$ & 3 & 1 & Yes \\
\hline
\end{tabular}

handle such messages, but $\mathrm{CMC}$ is strictly sequential; $\mathrm{EME}^{*}$ requires one key from $\mathcal{K}$ plus two other $n$-bit keys; and HCTR requires one key from $\mathcal{K}$ and one $n$-bit key. On the other hand, EME uses a single key and is fully parallel but can handle at most $n$ many $n$-bit blocks.

A general comparison of the computation cost of PEP and HCTR with the other modes (CMC, EME and $\mathrm{EME}^{*}$ ) is difficult. This is because in one case we have less block cipher invocations but $G F\left(2^{n}\right)$ multiplications whereas in the other case we have more block cipher invocations and no $G F\left(2^{n}\right)$ multiplications. Consequently, the comparison depends upon several factors such as:

1. The implementation platform - hardware or software. For software implementation, we need to consider the target architecture and its support for $G F\left(2^{n}\right)$ multiplication. Availability of cryptographic co-processors may provide substantial support for such operation. For hardware implementation, the number of available parallel encryption units and $G F\left(2^{n}\right)$ multiplication units need to be considered.

2. The most important consideration is the design of the actual block cipher being used with the mode of operation and its efficient implementation.

Note that PEP approximately trades one block cipher call for four multiplications (HCTR trades one $[\mathrm{BC}]$ for two $[\mathrm{M}]$ ). Implementation results from [10, suggest that using a look-up table it is possible to complete a few $G F\left(2^{n}\right)$ multiplications in the time required for one AES-128 invocation. A more detailed software speed comparison requires efficient implementation of the various modes of operation and can be a topic of future study.

A mode of operation is not designed for use with any particular block cipher. As a side remark, we would like to note that the FIPS 197 specifies AES for protecting "sensitive (unclassified) information". So each government might be having its own block cipher for protecting classified information. The computation cost of PEP vis-a-vis the other modes with respect to such ciphers has to be determined on a case-to-case basis.

The number of passes made by PEP and HCTR is one more than the other modes (though the number of encryption layers is one less). We do not consider 
this to be a serious problem since all the modes require at least two passes and cannot perform online encryption and decryption. The additional pass of PEP and HCTR by itself does not by itself lead to any efficiency degradation.

\section{Security of PEP}

\subsection{Definitions and Notation}

As mentioned earlier, an $n$-bit block cipher is a function $E: \mathcal{K} \times\{0,1\}^{n} \rightarrow$ $\{0,1\}^{n}$, where $\mathcal{K} \neq \emptyset$ is called the key space and for any $K \in \mathcal{K}, E(K,$.$) is a$ permutation. We will usually write $E_{K}()$ instead of $E(K,$.$) .$

An adversary $A$ is a probabilistic algorithm which has access to some oracles and which outputs either 0 or 1 . Oracles are written as superscripts. The notation $A^{\mathcal{O}_{1}, \mathcal{O}_{2}} \Rightarrow 1$ denotes the event that the adversary $A$, interacting with the oracles $\mathcal{O}_{1}, \mathcal{O}_{2}$, finally outputs the bit 1 .

Let $\operatorname{Perm}(n)$ denote the set of all permutations on $\{0,1\}^{n}$. We require $E($, to be a strong pseudorandom permutation. The advantage of an adversary in breaking the strong pseudorandomness of $E($,$) is defined in the following$ manner.

$$
\begin{aligned}
\operatorname{Adv}_{E}^{ \pm \operatorname{prp}}(A)= & \operatorname{Pr}\left[K \stackrel{\$}{\leftarrow} \mathcal{K}: A^{E_{K}(), E_{K}^{-1}()} \Rightarrow 1\right]- \\
& \operatorname{Pr}\left[\pi \stackrel{\$}{\leftarrow} \operatorname{Perm}(n): A^{\pi(), \pi^{-1}()} \Rightarrow 1\right] .
\end{aligned}
$$

Formally, a tweakable enciphering scheme is a function $\mathbf{E}: \mathcal{K} \times \mathcal{T} \times \mathcal{M} \rightarrow \mathcal{M}$, where $\mathcal{K} \neq \emptyset$ and $\mathcal{T} \neq \emptyset$ are the key space and the tweak space respectively and $\mathcal{M}=\cup_{i>1}\{0,1\}^{n i}$, where $n$ is the length of a message block. We shall often write $\mathbf{E}_{K}^{T}$ (.) instead of $\mathbf{E}(K, T,$.$) . The inverse of an enciphering scheme is \mathbf{D}=\mathbf{E}^{-1}$ where $X=\mathbf{D}_{K}^{T}(Y)$ if and only if $\mathbf{E}_{K}^{T}(X)=Y$.

Let $\operatorname{Perm}^{\mathcal{T}}(\mathcal{M})$ denote the set of all functions $\pi: \mathcal{T} \times \mathcal{M} \rightarrow \mathcal{M}$ where $\boldsymbol{\pi}(\mathcal{T},$. is a length preserving permutation. Such a $\pi \in \operatorname{Perm}^{\mathcal{T}}(\mathcal{M})$ is called a tweak indexed permutation. For a tweakable enciphering scheme $\mathbf{E}: \mathcal{K} \times \mathcal{T} \times \mathcal{M} \rightarrow$ $\mathcal{M}$, we define the advantage an adversary $A$ has in distinguishing $\mathbf{E}$ and its inverse from a random tweak indexed permutation and its inverse in the following manner.

$$
\begin{aligned}
& \mathbf{A d v}_{\mathbf{E}}^{ \pm \widetilde{\operatorname{prp}}}(A)=\operatorname{Pr}\left[K \stackrel{\$}{\leftarrow} \mathcal{K}: A^{\mathbf{E}_{K}(., .), \mathbf{E}_{K}^{-1}(., .)} \Rightarrow 1\right]- \\
& \operatorname{Pr}\left[\pi \stackrel{\$}{\leftarrow} \operatorname{Perm}^{\mathcal{T}}(\mathcal{M}): A^{\pi(., .), \pi^{-1}(., .)} \Rightarrow 1\right] .
\end{aligned}
$$

Pointless queries: An adversary never queries its deciphering oracle with $(T, C)$ if it got $C$ in response to an encipher query $(T, M)$ for some $M$. Similarly, the adversary never queries its enciphering oracle with $(T, M)$ if it got $M$ as a response to a decipher query of $(T, C)$ for some $C$. These queries are called pointless as the adversary knows what it would get as response for such queries. 
Following [5], we define the query complexity $\sigma_{n}$ of an adversary as follows. A string $X$ contributes $\max (|X| / n, 1)$ to the query complexity. A tuple of strings $\left(X_{1}, X_{2}, \ldots\right)$ contributes the sum of the contributions from all oracle queries plus the contribution from the adversary's output. Suppose an adversary makes $q$ queries where the number of $n$-bit blocks in the $i$ th query is $\ell_{i}$. Then, $\sigma_{n}=$ $1+\sum_{i=1}^{q}\left(1+\ell_{i}\right) \geq 2 q$. Let $\rho$ be a list of resources used by the adversary $A$ and suppose $\mathbf{A d v}_{\pi}^{ \pm x x x}(A)$ has been defined where $\pi$ is either a block cipher or a tweakable enciphering scheme. $\mathbf{A d v}_{\pi}^{ \pm \mathrm{xxx}}(\rho)$ denotes the maximal value of $\mathbf{A d v}_{\pi}^{ \pm x x x}(A)$ over all adversaries $A$ using resources at most $\rho$. Usual resources of interest are the running time $t$ of the adversary, the number of oracle queries $q$ made by the adversary and the query complexity $\sigma_{n}(n \geq 1)$.

The notation PEP $[E]$ denotes a tweakable enciphering scheme, where the $n$ bit block cipher $E$ is used in the manner specified by PEP. Our purpose is to show that $\mathrm{PEP}[E]$ is secure if $E$ is secure. The notation $\operatorname{PEP}[\operatorname{Perm}(n)]$ denotes a tweakable enciphering scheme obtained by plugging in a random permutation from Perm $(n)$ into the structure of PEP. For an adversary attacking $\operatorname{PEP}[\operatorname{Perm}(n)]$, we do not put any bound on the running time of the adversary, though we still put a bound on the query complexity $\sigma_{n}$. We show the information theoretic security of $\operatorname{PEP}[\operatorname{Perm}(n)]$ by obtaining an upper bound on $\mathbf{A d} \mathbf{d v}_{\operatorname{PEP}[\operatorname{Perm}(n)]}^{ \pm \widetilde{\operatorname{prp}}}\left(q, \sigma_{n}\right)$. The upper bound is obtained in terms of $n$ and $\sigma_{n}$. For a fixed block cipher $E$, we bound $\mathbf{A d v}_{\operatorname{PEP}[E]}^{ \pm \widetilde{\operatorname{prp}}}\left(q, \sigma_{n}, t\right)$ in terms of $\mathbf{A d v}_{E}^{ \pm \operatorname{prp}}\left(q, \sigma_{n}, t^{\prime}\right)$, where $t^{\prime}=t+O\left(\sigma_{n}\right)$. We will use the notation $\mathbf{E}_{\pi}$ as a shorthand for $\operatorname{PEP}[\operatorname{Perm}(n)]$ and $\mathbf{D}_{\pi}$ will denote the inverse of $\mathbf{E}_{\pi}$. Thus, the notation $A^{\mathbf{E}_{\pi}, \mathbf{D}_{\pi}}$ will denote an adversary interacting with the oracles $\mathbf{E}_{\pi}$ and $\mathbf{D}_{\pi}$.

\subsection{Statement of Result}

The following theorem specifies the security of PEP.

Theorem 1. Fix $n, q$ and $\sigma_{n} \geq q$ to be positive integers and an $n$-bit block cipher $E: \mathcal{K} \times\{0,1\}^{n} \rightarrow\{0,1\}^{n}$. Then

$$
\begin{gathered}
\mathbf{A d v}_{P E P[\operatorname{Perm}(n)]}^{ \pm \widetilde{p r p}}\left(q, \sigma_{n}\right) \leq \frac{1}{2^{n+1}} \times\left(q^{2}+6\left(5 q+\sigma_{n}\right)^{2}\right) \\
\mathbf{A d v}_{P E P[E]}^{ \pm \widetilde{p r p}}\left(q, \sigma_{n}, t\right) \leq \frac{1}{2^{n+1}} \times\left(q^{2}+6\left(5 q+\sigma_{n}\right)^{2}\right)+\mathbf{A d v}_{E}^{ \pm p r p}\left(q, \sigma_{n}, t^{\prime}\right)
\end{gathered}
$$

where $t^{\prime}=t+O\left(\sigma_{n}\right)$.

Since each query consists of at least one $n$-bit block, we have $q \leq \sigma_{n}$ and hence we could write $\left(q^{2}+6\left(5 q+\sigma_{n}\right)^{2}\right) \leq c \sigma_{n}^{2}$ for some constant $c$. Upper bounding $q$ by $\sigma_{n}$ is proper when $\sigma_{n}$ and $q$ are comparable, i.e., when each query consists of a few blocks. On the other hand, if each query consists of a large number of blocks, the bound $q \leq \sigma_{n}$ is very loose and replacing $q$ by $\sigma_{n}$ makes the bound appear worse than what it really is. Hence, we choose to present the bound in terms of both $q$ and $\sigma_{n}$. 
The above result and its proof is similar to previous work (see for example [4514]). As mentioned in [4], Equation (5]) embodies a standard way to pass from the information theoretic setting to the complexity theoretic setting. Let $\mathbf{E}(., .,$.$) denote \operatorname{PEP}[E]$. For any adversary $A$, we have the following.

$$
\begin{aligned}
& \mathbf{A d v}_{\operatorname{PEP}[E]}^{ \pm \widetilde{\operatorname{prp}}}(A) \\
& =\operatorname{Pr}\left[K \stackrel{\$}{\leftarrow} \mathcal{K}: A^{\mathbf{E}_{K}(., .), \mathbf{E}_{K}^{-1}(., .)} \Rightarrow 1\right]-\operatorname{Pr}\left[\boldsymbol{\pi} \stackrel{\$}{\leftarrow} \operatorname{Perm}^{\mathcal{T}}(\mathcal{M}): A^{\boldsymbol{\pi}(., .), \boldsymbol{\pi}^{-1}(., .)} \Rightarrow 1\right] \\
& =\left(\operatorname{Pr}\left[K \stackrel{\$}{\leftarrow} \mathcal{K}: A^{\mathbf{E}_{K}(., .), \mathbf{E}_{K}^{-1}(., .)} \Rightarrow 1\right]-\operatorname{Pr}\left[\pi \stackrel{\$}{\leftarrow} \operatorname{Perm}(n): A^{\mathbf{E}_{\pi}, \mathbf{D}_{\pi}} \Rightarrow 1\right]\right)+ \\
& \operatorname{Pr}\left[\pi \stackrel{\$}{\leftarrow} \operatorname{Perm}(n): A^{\mathbf{E}_{\pi}, \mathbf{D}_{\pi}} \Rightarrow 1\right]-\operatorname{Pr}\left[\boldsymbol{\pi} \stackrel{\$}{\leftarrow} \operatorname{Perm}^{\mathcal{T}}(\mathcal{M}): A^{\boldsymbol{\pi}(., .), \boldsymbol{\pi}^{-1}(. . .)} \Rightarrow 1\right] \\
& =X+\mathbf{A d} \mathbf{v}_{\operatorname{PEP}[\operatorname{Perm}(n)]}^{ \pm \widetilde{\operatorname{prp}}}(A)
\end{aligned}
$$

where

$$
X=\left(\operatorname{Pr}\left[K \stackrel{\$}{\leftarrow} \mathcal{K}: A^{\mathbf{E}_{K}(., .), \mathbf{E}_{K}^{-1}(., .)} \Rightarrow 1\right]-\operatorname{Pr}\left[\pi \stackrel{\$}{\leftarrow} \operatorname{Perm}(n): A^{\mathbf{E}_{\pi}, \mathbf{D}_{\pi}} \Rightarrow 1\right]\right)
$$

The quantity $X$ represents an adversary's advantage in distinguishing $\mathrm{PEP}[E]$ from $\operatorname{PEP}[\pi]$, where $\pi$ is a randomly chosen permutation from $\operatorname{Perm}(n)$. Clearly, such an adversary $A$ can also distinguish $E$ from a random permutation and hence $X \leq \mathbf{A d v}_{E}^{ \pm \operatorname{prp}}(A)$. This argument shows how (4) is obtained from (5).

We need to consider an adversary's advantage in distinguishing a tweakable enciphering scheme $\mathbf{E}$ from an oracle which simply returns random bit strings. This advantage is defined in the following manner.

$$
\mathbf{A d v}_{\operatorname{MEM}[\operatorname{Perm}(n)]}^{ \pm \operatorname{rnd}}(A)=\operatorname{Pr}\left[\pi \stackrel{\$}{\leftarrow} \operatorname{Perm}[n]: A^{\mathbf{E}_{\pi}, \mathbf{D}_{\pi}} \Rightarrow 1\right]-\operatorname{Pr}\left[A^{\$(., .), \$(., .)} \Rightarrow 1\right]
$$

where $\$(.,$.$) returns random bits of length |M|$. The basic idea of proving (4) is as follows.

$$
\begin{aligned}
& \mathbf{A d v}_{\operatorname{PEP}[\operatorname{Perm}(n)]}^{ \pm \widetilde{\operatorname{Prp}}}(A) \\
= & \operatorname{Pr}\left[\pi \stackrel{\$}{\leftarrow} \operatorname{Perm}(n): A^{\mathbf{E}_{\pi}, \mathbf{D}_{\pi}} \Rightarrow 1\right]-\operatorname{Pr}\left[\boldsymbol{\pi} \stackrel{\$}{\leftarrow} \operatorname{Perm}^{\mathcal{T}}(\mathcal{M}): A^{\boldsymbol{\pi}(., .), \boldsymbol{\pi}^{-1}(., .)} \Rightarrow 1\right] \\
= & \left(\operatorname{Pr}\left[\pi \stackrel{\$}{\leftarrow} \operatorname{Perm}(n): A^{\mathbf{E}_{\pi}, \mathbf{D}_{\pi}} \Rightarrow 1\right]-\operatorname{Pr}\left[A^{\$(., .), \$(., .)} \Rightarrow 1\right]\right)+ \\
& \left(\operatorname{Pr}\left[A^{\$(., .), \$(., .)} \Rightarrow 1\right]-\operatorname{Pr}\left[\boldsymbol{\pi} \stackrel{\$}{\leftarrow} \operatorname{Perm}^{\mathcal{T}}(\mathcal{M}): A^{\boldsymbol{\pi}(., .), \boldsymbol{\pi}^{-1}(., .)} \Rightarrow 1\right]\right) \\
\leq & \operatorname{Adv}_{\operatorname{PEP}[\operatorname{Perm}(n)]}^{ \pm \operatorname{rnd}}(A)+\left(\begin{array}{l}
q \\
2
\end{array}\right) \frac{1}{2^{n}}
\end{aligned}
$$

where $q$ is the number of queries made by the adversary. For a proof of the last inequality see [5].

The main task of the proof now reduces to obtaining an upper bound on $\mathbf{A d v} \mathbf{P E P}_{\operatorname{Perm}(n)]}^{ \pm \operatorname{rnd}}\left(\sigma_{n}\right)$. The complete proof is provided in [2]. The proof uses the 
standard technique of sequence of games between an adversary and the mode of operation PEP. The proof is similar to the corresponding proofs of CMC [4] and EME [5. By a sequence of games we show that if in response to any valid query of the adversary, random strings of appropriate lengths are returned then the internal computations of PEP can be performed consistently under the assumption that the block cipher and its inverse are random permutations. The crux of the proof lies in showing that there would seldom be any collisions in the range and domain sets of the block cipher if the adversary queries PEP with valid queries and PEP responds to them by producing random strings. In a later part we remove the randomness associated with the adversary and the game runs on a fixed transcript consisting of the queries and their responses. We show that in such a situation also the internal computations of PEP can be performed consistently.

We later prove (see [2]) that for any adversary making $q$ queries and having query complexity $\sigma_{n}$

$$
\mathbf{A d v}_{\operatorname{PEP}[\operatorname{Perm}(n)]}^{ \pm \operatorname{rnd}}\left(q, \sigma_{n}\right) \leq \frac{3\left(5 q+\sigma_{n}\right)^{2}}{2^{n}} .
$$

Using this and (6), we obtain

$$
\mathbf{A d v}_{\operatorname{PEP}[\operatorname{Perm}(n)]}^{\widetilde{\operatorname{prp}}}\left(q, \sigma_{n}\right) \leq \frac{1}{2^{n+1}} \times\left(q^{2}+6\left(5 q+\sigma_{n}\right)^{2}\right) .
$$

\section{Conclusion}

We have presented a new construction for a tweakable SPRP called PEP. Our approach is to use polynomial hash, followed by an encryption layer and again followed by a polynomial hash. This is different from the other constructions of tweakable SPRPs, namely CMC, EME and EME* and is similar to the approach for constructing SPRP (not tweakable SPRP) given in 11]; this approach has also been used in constructing HCTR. PEP offers certain advantages over the known tweakable SPRPs - it is the only know construction which uses a single key, is efficiently parallelizable and can handle an arbitrary number of blocks. We make a detailed comparison between the known constructions of tweakable SPRPs which show that PEP compares quite favorably

\section{Acknowledgement}

We would like to thank Peng Wang for pointing out two errors in a previous version of this paper which had specified a different mode of operation.

\section{References}

1. M. Bellare and P. Rogaway, Encode-then-encipher encryption: How to exploit nonces or redundancy in plaintexts for efficient cryptography, Advances in Cryptology - Asiacrypt 2000, LNCS 1976, pp. 317-330, Springer, 2000. 
2. D. Chakraborty and P. Sarkar, A new mode of encryption providing a tweakable strong pseudorandom permutation, eprint.iacr.org, 2006

3. S. Halevi, EME* . Extending EME to handle arbitrary-length messages with associated data. INDOCRYPT 2004, pp. 315-327, Springer 2004

4. S. Halevi and P. Rogaway. A tweakable enciphering mode, Advances in Cryptology - CRYPTO 2003, LNCS, vol. 2729, pp. 482-499, Springer, 2003.

5. S. Halevi and P. Rogaway. A parallelizable enciphering mode, Topics in Cryptology, CT-RSA 2004, LNCS, vol. 2964, pp. 292-304, Springer, 2004

6. C. S. Jutla: Encryption modes with almost free message integrity. EUROCRYPT 2001: 529-544.

7. S. Maitra, K. C. Gupta and A. Venkateswarlu. Results on multiples of primitive polynomials and their products over $G F(2)$. Theoretical Computer Science 341(13): 311-343 (2005).

8. M. Liskov, R. L. Rivest and D. Wagner. Tweakable block ciphers. CRYPTO 2002: $31-46$.

9. M. Luby and C. Rackoff, How to construct pseudo-random permutations and pseudo-random functions, SIAM Journal of Computing, vol. 17, pp. 373-386, 1988.

10. D. A. McGrew and J. Viega. The Security and Performance of the Galois/Counter Mode (GCM) of Operation. Proceedings of Indocrypt 2004, 343-355.

11. M. Naor and O. Reingold. On the construction of pseudo-random permutations: Luby-Rackoff revisited, J. of Cryptology, vol 12, pp. 29-66, 1999.

12. M. Naor and O. Reingold. A pseudo-random encryption mode. Manuscript available from www.wisdom.weizmann.ac.il/naor.

13. P. Rogaway. Nonce-based symmetric encryption, Fast Software Encryption (FSE) 2004, LNCS 3017, pp. 348-359, Springer, 2004.

14. P. Rogaway, M. Bellare and J. Black. OCB: A block-cipher mode of operation for efficient authenticated encryption. ACM Conference on Computer and Communication Security 2001: 196-205.

15. D. Wagner. A generalized birthday problem. CRYPTO 2002, LNCS 2442, pp. 288 303, Springer 2002.

16. M. Wegman and L. Carter. New hash functions and their use in authentication and set equality. Journal of Computer and System Sciences, vol. 22, 1981, pp. 265-279.

17. P. Wang, D. Feng and W. Wu. HCTR: A variable-input-length enciphering mode. CISC 2005, LNCS 3822, pp. 175-188, 2005. 